\title{
Important lessons for infection control in radiology departments during the COVID-19 pandemic
}

\author{
Anand Devaraj ${ }^{1}$ \\ Received: 31 March 2020 / Accepted: 7 April 2020 / Published online: 1 May 2020 \\ (C) European Society of Radiology 2020
}

Radiologists worldwide have benefited from the considerable volume of literature on the imaging appearances of COVID19 pneumonia that has emerged from high-incidence centres in China.

In the latest online publications of European Radiology, Gutzeit et al [1], Deng et al [2] and Politi et al [3] have produced guidance on the critically important subject of infection control in radiology departments. The guidance covers areas such as personal protective equipment, cleaning protocols, and waste disposal. All three articles stress the importance of staff training as well as the need for identifying key personnel with responsibilities for infection control specifically within the radiology department. The authors also emphasize the need for establishing "clean" and "infected" zones in hospitals, including separate CT scanners with dedicated transfer routes for patients with confirmed COVID-19 pneumonia. This may be challenging logistically for some institutions. Scanning consecutive patients with COVID-19 pneumonia in batches, within pre-specified timeslots, may be a suitable alternative approach. Some aspects of the guidance will not be readily transferrable to all institutions and countries, such as the recommendation for dedicated isolation and observation wards for clinical staff, following work in high COVID-19 intensity wards.

Another important area to consider, which is not covered in so much detail, is developing protocols for patient transfer to CT scanners, in particular from ICU units, including for example specific guidance for radiographic and ICU staff during the scanning process. Nevertheless, these articles, in conjunction with other existing literature [4], will be instructive for readers looking to develop or refine local radiology-specific infection control guidelines.

This comment refers to the articles available at https://doi.org/10.1007/ s00330-020-06841-6, https://doi.org/10.1007/s00330-020-06850-5, and https://doi.org/10.1007/s00330-020-06871-0.

Anand Devaraj

a.devaraj@nhs.net

1 Department of Radiology, Royal Brompton Hospital, London, UK
Funding information The authors state that this work has not received any funding.

\section{Compliance with ethical standards}

Guarantor The scientific guarantor of this publication is Anand Devaraj.

Conflict of interest The authors of this manuscript declare no relationships with any companies whose products or services may be related to the subject matter of the article.

Statistics and biometry No complex statistical methods were necessary for this paper.

Informed consent Written informed consent was not required for this study because it is an Editorial.

Ethical approval Institutional Review Board approval was not required because this work is an Editorial.

Methodology

- This work is an Editorial.

\section{References}

1. Gutzeit A, Li Q, Matoori S, Li B, Wang L (2020) What can European radiologists learn from the outbreak of COVID-19 in China? A discussion with a radiologist from Wuhan. Eur Radiol. https://doi.org/ 10.1007/s00330-020-06841-6

2. Ding J, Fu H, Liu Y et al (2020) Prevention and control measures in radiology department for COVID-19. Eur Radiol. https://doi.org/10. $1007 / \mathrm{s} 00330-020-06850-5$

3. Politi LS, Balzarini L (2020) The radiology department during the COVID-19 pandemic: a challenging, radical change. Eur Radiol. https://doi.org/10.1007/s00330-020-06871-0

4. COVID-19 Guidance for infection prevention and control in healthcare settings: Issued jointly by the Department of Health and Social Care (DHSC), Public Health Wales (PHW), Public Health Agency (PHA) Northern Ireland, Health Protection Scotland (HPS) and Public Health England as official guidance. https://assets.publishing.service.gov.uk/ government/uploads/system/uploads/attachment_data/file/881489/ COVID-19_Infection_prevention_and_control_guidance_complete.pdf. Accessed 28 Mar 2020

Publisher's note Springer Nature remains neutral with regard to jurisdictional claims in published maps and institutional affiliations. 